Letter to the editor

\section{Gender differences in motives for physical activity across the stages of change in Ugandan outpatients with psychosis}

The biopsychosocial benefits of physical activity (PA) for people with psychosis are nowadays well established (Firth et al., 2015). Translation and integration of these research findings from clinical trials to routine care is urgently required. Motivating people with psychosis to adopt an active lifestyle is however challenging (Vancampfort et al., 2015). There is a clear need for theoretical evidence that might assist health care professionals in facilitating this motivational process. Research from a highincome country (Belgium), where physical activity is an important aspect of the multidisciplinary treatment, demonstrated that there is preliminary evidence for the self-determination theory (SDT) (Deci and Ryan, 2000) in understanding PA and exercise adoption among people with psychosis (Vancampfort et al., 2014). This research highlighted the importance of considering identified and integrated motivations (i.e. perceiving PA outcomes as personally important) and intrinsic motivations (i.e. perceiving PA as challenging, enjoyable and/or rewarding) in both men and women (Vancampfort et al., 2014). However, these data are yet to be confirmed in other settings throughout the world, and it is unknown whether culture-sensitive gender differences need to be considered. For example, for many women in African societies, there is a taboo attached to weight-loss following PA, which is often viewed as related to being poor or having HIV/AIDS (Kinsman et al., 2014). We investigated whether (1) SDT can explain physical activity behaviors in a low resource setting in Uganda, a low-income country where physical activity is not an aspect of the multidisciplinary treatment of psychosis (Vancampfort et al., 2017), and (2) if motivation towards PA differs across genders. The aim was to explore whether the motivational regulations differed across different stages of behavior change (Prochaska and DiClemente, 1992), taking into account gender differences. Over a 3-month period, 24 male ( $34.8 \pm 10.6$ years) and 24 female (31.9 \pm 8.4 years) outpatients with a DSM 5 diagnosis of any psychotic disorder, as diagnosed by the treating psychiatrist of the Butabika National Referral Hospital, Kampala, Uganda completed the Behavioral Regulation Questionnaire - 3 (BREQ-3) (Markland and Tobin, 2004) and the Patient-centred Assessment and Counselling for Exercise questionnaire (Long et al., 1996). A multivariate analysis of variance (MANOVA) was used to test for significant differences in BREQ-3 subscale scores across the TTM stages of change and between men and women. Where significant main effects were demonstrated, we followed up with comparisons of differences across stages of change groups, using one-way analyses of variance (ANOVA) with post hoc tests (Scheffe). Significance level was set at $p<0.05$. SPSS 24.0 was used for data analysis (SPSS Inc., Chicago, IL). The study procedure was approved by the ethical committee of Mengo Hospital. All participants provided written informed consent. Our data show that people with psychosis in the maintenance stage had higher identified, integrated and intrinsic regulation scores than those in the (pre) preparation stage. There were gender differences. Women in the (pre-)preparation stage had higher levels of a-motivation than those in the action stage. Among men, higher identified, integrated and intrinsic regulation scores were observed for those in the maintenance versus (pre-) preparation stage (see Table 1). Men also had significantly higher introjected regulation scores than women $(\mathrm{P}=0.048)$ (see Table 1$)$. Thus, our data demonstrate that, similarly to research in high-income countries, SDT (Vancampfort et al., 2013) and the stages of change model (Vancampfort et al., 2014) can be used in order to underpin strategies for motivating people with psychotic disorders to an active lifestyle in a low resource non-Western setting. Our data are the first to demonstrate that culture-sensitive gender differences should be considered. Only in men higher identified, integrated and intrinsic regulation scores were observed for those in the maintenance versus the (pre-) preparation stage. This shows that in men autonomous regulations are not only important for adopting but also for maintaining exercise behaviors over a longer period (i.e. $>6$ months). In contrast, among women, a-motivation explained differences between those who were and were not physically active ( $<6$ months). As this is the first study to investigate gender differences in motivation towards PA across the different stages of change in a non-Western population, our findings have clear clinical implications. Specifically, health care professionals should consider gender differences when motivating people with psychotic disorders in order

Table 1

Means and standard deviations and differences in the BREQ-3 subscales by stage of change in Ugandan outpatients with psychosis $(\mathrm{n}=48)$.

\begin{tabular}{|c|c|c|c|c|c|c|c|c|}
\hline \multirow[t]{2}{*}{ Motivational type } & \multicolumn{2}{|c|}{$\begin{array}{l}\text { (Pre-) } \\
\text { preparation } \\
(\mathrm{n}=17)\end{array}$} & \multicolumn{2}{|c|}{$\begin{array}{l}\text { Action } \\
(\mathrm{n}=14)\end{array}$} & \multicolumn{2}{|c|}{$\begin{array}{l}\text { Maintenance } \\
(\mathrm{n}=17)\end{array}$} & \multirow[t]{2}{*}{ F-value } & \multirow[t]{2}{*}{ p-Value } \\
\hline & M & SD & M & SD & M & SD & & \\
\hline A-motivation & 1.6 & 0.6 & 1.9 & 0.7 & 1.6 & 0.5 & 2.03 & 0.14 \\
\hline Men & 1.6 & 0.7 & 1.6 & 0.7 & 1.5 & 0.5 & 0.5 & 0.95 \\
\hline Women & $1.6^{\mathrm{a}}$ & 0.5 & $2.2^{\mathrm{a}}$ & 0.6 & 1.8 & 0.6 & 4.5 & $0.02^{*}$ \\
\hline External regulation & 1.7 & 0.8 & 2.3 & 0.6 & 1.8 & 1.0 & 2.2 & 0.12 \\
\hline Men & 2.1 & 0.8 & 2.4 & 0.8 & 2.0 & 1.1 & 0.6 & 0.57 \\
\hline Women & 1.4 & 0.6 & 2.2 & 0.4 & 1.7 & 1.1 & 1.7 & 0.21 \\
\hline Introjected regulation & 1.7 & 0.8 & 2.2 & 0.7 & 2.5 & 1.1 & 3.1 & 0.06 \\
\hline Men & $1.9^{\circ}$ & 0.9 & 2.4 & 0.8 & 2.8 & 1.2 & 2.1 & 0.14 \\
\hline Women & $1.6^{\circ}$ & 0.8 & 2.0 & 0.6 & 2.1 & 0.8 & 1.2 & 0.32 \\
\hline Identified regulation & $2.4^{\mathrm{b}}$ & 0.7 & 2.6 & 0.6 & $3.0^{\mathrm{b}}$ & 0.6 & 4.0 & $0.02^{*}$ \\
\hline Men & $2.1^{\mathrm{b}}$ & 0.7 & 2.7 & 0.7 & $3.3^{\mathrm{b}}$ & 0.3 & 7.8 & $0.003^{*}$ \\
\hline Women & 2.7 & 0.5 & 2.5 & 0.6 & 2.7 & 0.6 & 0.5 & 0.62 \\
\hline Integrated regulation & $2.1^{b}$ & 0.8 & 2.7 & 0.8 & $2.9^{\mathrm{b}}$ & 0.7 & 4.8 & $0.01^{*}$ \\
\hline Men & $2.1^{b}$ & 0.8 & 2.8 & 1.0 & $3.3^{\mathrm{b}}$ & 0.4 & 4.3 & $0.03^{*}$ \\
\hline Women & 2.0 & 0.9 & 2.6 & 0.6 & 2.5 & 0.8 & 1.31 & 0.29 \\
\hline Intrinsic regulation & $2.2^{\mathrm{b}}$ & 0.6 & 2.6 & 0.7 & $3.0^{\mathrm{b}}$ & 0.9 & 4.6 & $0.02^{*}$ \\
\hline Men & $2.2^{\mathrm{b}}$ & 0.5 & 2.7 & 0.7 & $3.4^{\mathrm{b}}$ & 0.6 & 6.6 & $0.006^{*}$ \\
\hline Women & 2.3 & 0.6 & 2.4 & 0.5 & 2.4 & 0.8 & 0.6 & 0.54 \\
\hline
\end{tabular}

$\overline{\mathrm{M}}=$ mean, $\mathrm{SD}=$ standard deviation, MANOVA ( ${ }^{*}$ model significant at $\left.\mathrm{p}<0.05\right)$ with post hoc Scheffe when indicated (significance set here at $\mathrm{p}<0.05$ ): $\mathrm{b}=$ (pre-)preparation versus maintenance. ${ }^{\circ}$ Significant gender differences $(\mathrm{P}<0.05)$. 
to become more physically active. While in men in particular autonomous regulations should be stimulated, in women a-motivation should be reduced. In conclusion, our study indicates that the self-determination theory and the stages of change theory are transcultural models. However, health care professionals should consider culture-sensitive gender differences in the promotion of PA.

\section{Contributors}

We certify that all authors have contributed significantly, and that all authors are in agreement with the content of the manuscript. More in detail, the study was designed by Davy Vancampfort, Michel Probst, Tine Van Damme and James Mugisha. All data were collected by Davy Vancampfort and James Mugisha. Statistical analyses were performed by Michel Probst. Davy Vancampfort wrote the first draft of the paper, all other co-authors commented and contributed to the subsequent revisions. All authors have approved the final manuscript.

\section{Conflict of interest}

All authors declare that they have no conflicts of interest related to this study. We also certify that no party having a direct interest in the results of the research supporting this article has or will confer a benefit on us or on any organization with which we are associated.

\section{Acknowledgements}

None to report.

\section{References}

Deci, E.L., Ryan, R.M., 2000. The "what" and" why" of goal pursuits: human needs and the self- determination of behavior. Psychol. Inq. 11 (4), 227-268.

Firth, J., Cotter, J., Elliott, R., French, P., Yung, A., 2015. A systematic review and meta-analysis of exercise interventions in schizophrenia patients. Psychol. Med. 45 (7), 1343-1361.

Kinsman, J., Norris, S.A., Kahn, K., Twine, R., Riggle, K., Edin, K., Mathebula, J., Ngobeni, S. Monareng, N., Micklesfield, L.K., 2014. A model for promoting physical activity among rural South African adolescent girls. Glob. Health Action 8, 28790.

Long, B.J., Calfas, K.J., Wooten, W., Sallis, J.F., Patrick, K., Goldstein, M., Marcus, B.H. Schwenk, T.L., Chenoweth, J., Carter, R., 1996. A multisite field test of the acceptability of physical activity counseling in primary care: project PACE. Am. J. Prev. Med. 12 (2), 73-81.

Markland, D., Tobin, V., 2004. A modification to the behavioural regulation in exercise questionnaire to include an assessment of amotivation. J. Sport Exerc. Psychol. 26 (2), 191-196.

Prochaska, J.O., DiClemente, C.C., 1992. Stages of change in the modification of problem behaviors. Prog. Behav. Modif. 28, 183.

Vancampfort, D., De Hert, M., Vansteenkiste, M., De Herdt, A., Scheewe, T.W., Soundy, A., Stubbs, B., Probst, M., 2013. The importance of self-determined motivation towards physical activity in patients with schizophrenia. Psychiatry Research 210 (3), 812-818.
Vancampfort, D., Vansteenkiste, M., De Hert, M., De Herdt, A., Soundy, A., Stubbs, B., Buys, R., Probst, M., 2014. Self-determination and stage of readiness to change physical activity behaviour in schizophrenia. Ment. Health and Phys. Act. 7 (3), 171-176.

Vancampfort, D., Stubbs, B., Ward, P., Teasdale, S., Rosenbaum, S., 2015. Integrating physical activity as medicine in the care of people with severe mental illness. Aust. N. Z. J. Psychiatry 49 (8), 681-682.

Vancampfort, D., Stubbs, B., De Hert, M., du Plessis, C., Gbiri, C.A.O., Kibet, J., Wanyonyi, N. Mugisha, J., 2017. A systematic review of physical activity policy recommendations and interventions for people with mental health problems in sub-Saharan African countries. Pan Afr. Med. J. 26 (104).

Davy Vancampfort

KU Leuven - University of Leuven, Department of Rehabilitation Sciences, Leuven, Belgium

KU Leuven - University of Leuven, University Psychiatric Centre, LeuvenKortenberg, Belgium

Corresponding author at: Tervuursevest 101, 3001 Leuven, Belgium. E-mail address: davy.vancampfort@kuleuven.be

Michel Probst

KU Leuven - University of Leuven, Department of Rehabilitation Sciences, Leuven, Belgium

NICM School of Health and Science, University of Western Sydney, Australia

Division of Psychology and Mental Health, University of Manchester, UK

Simon Rosenbaum

School of Psychiatry, UNSW, Sydney, Australia Black Dog Institute, Prince of Wales Hospital, Sydney, Australia

Tine Van Damme

KU Leuven - University of Leuven, Department of Rehabilitation Sciences, Leuven, Belgium

James Mugisha

Kyambogo University, Kampala, Uganda Butabika National Referral and Mental Health Hospital, Kampala, Uganda

7 August 2017 Available online $\mathrm{xxxx}$ 